\title{
Methodology for Construction of a T-Pose 3D Human Model
}

\author{
Eric ENNIS*, Christopher HESS, Isiah DAVENPORT \\ anfoscitex Corporation, a DCS Company, Dayton OH, USA \\ http://dx.doi.org/10.15221/13.107
}

\begin{abstract}
Restrictions in the scan volume of typical 3D body scanners, such as the 3dMD Body Scanning System, have imposed severe limitations in their use for creation of 3D models for the purposes of human animation. For these purposes, the ideal pose is to have the human subject stand with the feet shoulder-width apart and arms held completely horizontal at the shoulders (known as a T-Pose). This pose, however, is difficult to capture, especially for taller individuals whose arms may not fit within the capture volume. As a result, scans are typically collected with arms down towards the body rather than parallel to the ground. With these scans, animators are forced to choose between creating a completely fictional human (e.g. an artist's rendition) or painstakingly modifying the scan to arrange the underlying 'bones' into the proper T-Pose. One type of 3D mesh file utilizes a process known as vertex mapped texturing, in which an RGB color value is assigned to each vertex in the mesh. Another method is known as texture mapping, in which a separate texture file is created that 'wraps around' a geometric mesh. This process usually leads to visually pleasing scans; however, some difficulties arise with these files. For instance, the texture mapping process makes it impossible to merge scan files together while retaining color/texture information. This paper explores a method of generating 3D mesh models in two major scan formats, through the use of merging multiple scans and a series of software operations to reduce the amount of time and effort required by animators/computer graphics professionals.
\end{abstract}

\section{Introduction}

When taking a 3D scan of a human subject, the resulting mesh file can be used in many ways. One example is to use the subject as an 'actor' in animation purposes. In this process, the subject scan is matched to an underlying skeleton (Fig. 1). The skeleton is then animated which results in corresponding animated movements in the subject scan.

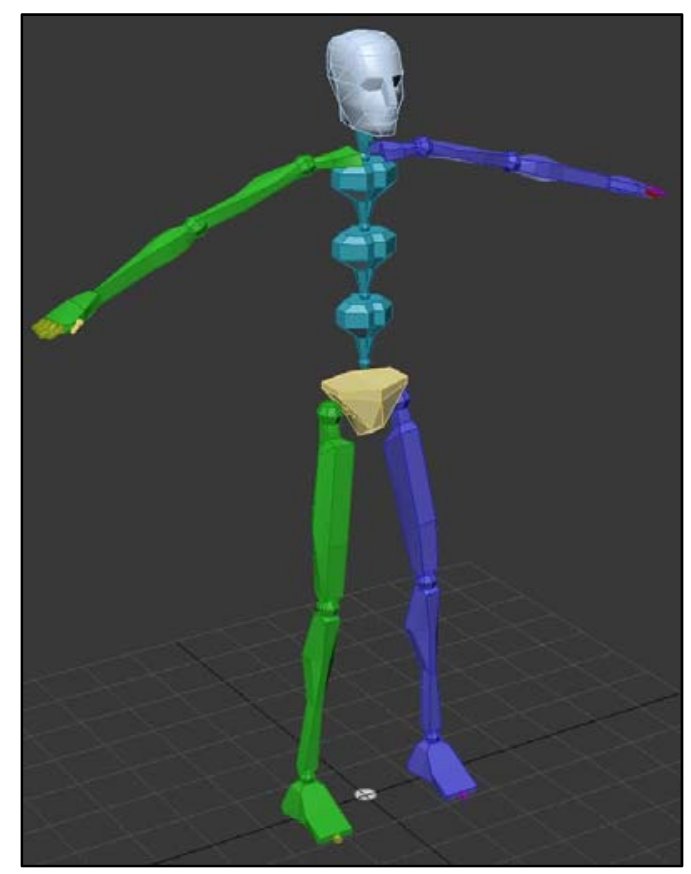

Fig. 1. Biped Skeleton (used for Animation).

*eennis@infoscitex.com 
For this mapping process, the skeleton is arranged in a pose with the feet shoulder-width apart and arms held completely horizontal at the shoulders (commonly referred to as a T-Pose). This introduces a problem in that most 3D body scanners do not possess a large enough scan volume to collect subjects in a T-Pose, particularly for the extended arms. For many researchers, the solution is to modify the pose of the subject at the time of scan to gain full visibility of the subject. This is done by moving the arms closer to, but not touching the body, so that the arms are distinct shapes that can still be captured in the volume.

A standard pose (Fig. 2) was defined in the Civilian American European Surface Anthropometric Resource (CAESAR) Survey [1]. However, scans in this configuration cause difficulties for computer graphics personnel when matching the individual's mesh to the animation skeleton since the poses are different. Additionally, most animation software packages require a specific 3D mesh format with an associated (but separate) image file, which maps to the underlying geometric mesh. Decoupled mapping causes a problem with the traditional methods that could be used to reconcile various scanned poses when they are mapped to an animation model. This paper will detail a process for reducing the time and effort required for animators in manually resolving these issues.

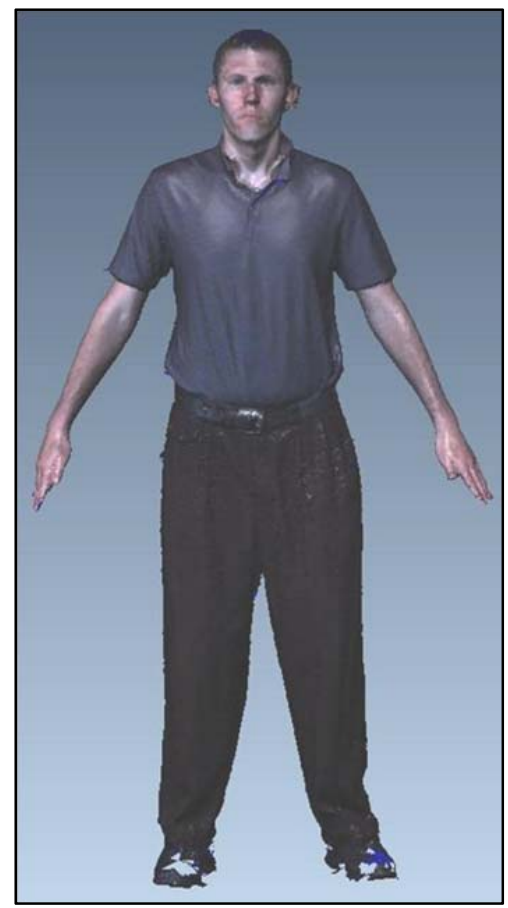

Fig. 2. Standard CAESAR Pose.

\section{Background}

Since the advent of 3D scanners designed for human data collection, a dilemma has been present regarding the trade-off between increasing scan volume (usually by moving stationary sensors further from the subject) and decreasing the level of detail for a scan. A good balance has been reached in most of today's scanners. This balance, however, often fails to capture the entire human when a pose is taken in which the subject's arms are spread wide. Typically researchers pose the subject in a non-standard T-pose in order to get the entire person in the scan volume. While some of the newest scanners on the commercial market available greatly increase the scan volume, these scanners often require a large number of cameras, and are quite expensive. Hand-held scanners are also an option, however, the time required for scans is typically quite long, which causes movement artifacts to be more pronounced. 


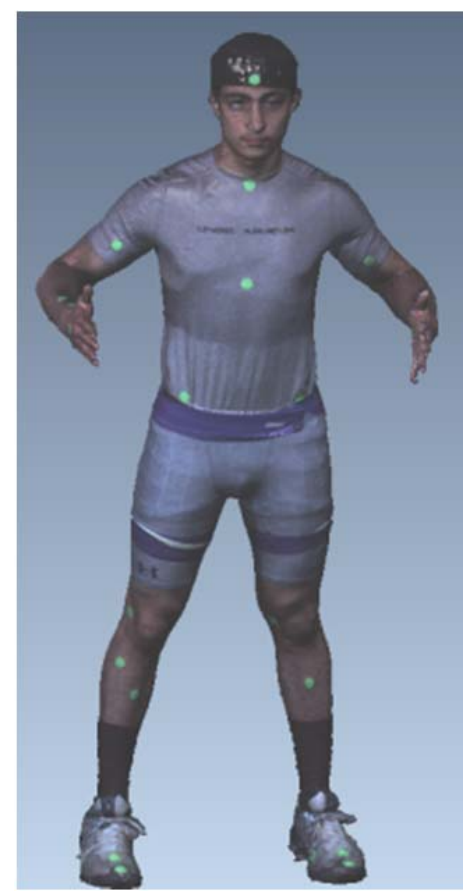

Fig. 3. Alternate CAESAR Pose.

In testing conducted by Infoscitex, two standard poses were used to scan individuals. The aforementioned, CAESAR pose and an alternate CAESAR pose (Fig. 3) in which the arms are horizontal beginning at the elbows. These poses can fit in most scanner capture volumes, but using them introduces new problems. In these poses, mesh files often have holes where the subject is selfoccluding the view from the sensor. Additionally, insufficient resolution can be present in areas where the limbs meet the torso. The underarms of many mesh files are often not well defined due to the arms being too close to the body. (Fig. 4) For the purpose of using a mesh file for animation, poor resolution in the underarm area causes problems that are unacceptable as activities where the animated model reaches overhead or outward, away from the body, look unrealistic. The animator is forced to become a 'body-artist' in these problem areas modifying the scan manually in order to correct the missing or poor resolution data. In the example of the under-arms, the animator must make a decision about where the arm ends and where the torso begins. When this is done, some of the advantages of using the 3D scan are lost, as ground-truth accuracy is replaced by an artist's interpretation of the subject.

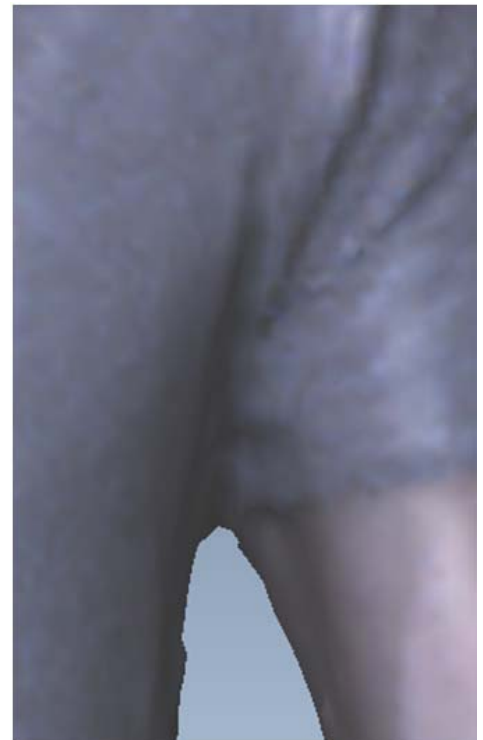

Fig. 4. Obscure data under subject's arms. 
An additional consideration of this paper is the format of the resulting mesh created from the scanner. There are two separate types of scan files. For this paper they are defined as 'texture-mapped' or 'vertex-mapped' files. Texture-mapped files include a geometry file as well as a separate image file, often a .bmp. When the scan file is opened, the image file is wrapped around the geometry of the scan. The image data is mapped to individual faces of the scan. By doing this, the models look realistic as they contain many different pixels of image data related to a single polygon. While the scan file looks better to the average viewer, the underlying geometry can be quite sparse. For this reason, animation software packages overwhelmingly require this format.

Vertex mapped files are meshes that contain the geometric points of a file with an XYZ value in addition to an RGB value. In this way the color is mapped onto each vertex. The advantage of this is that it allows for most software packages to interpolate color from nearby vertices when holes are filled. Additionally, the actual geometric point cloud tends to be more condensed, making for smoother surfaces with more accurate curvature values.

These issues highlighted a need to have a more encompassing subject capture to enable a more accurate scan file for animation and to reduce the processing time required by animators to create animation files.

\section{Process}

Laboratory personnel have developed a method to create a 3D mesh file of a subject oriented in a standing posture with the feet shoulder-width apart and arms held completely horizontal at the shoulders (known as a T-Pose). This pose is the standard for animation purposes and would be helpful for other data uses as well. The process undertaken utilizes the 3dMD Body Scanning System, though it can be replicated with most any scanner. Most configurations of this scanner attempting to get high resolution scans do not have a large enough volume to capture the full volume of a subject's arms in the T-Pose. However, the scanner's high resolution capture capabilities along with open source software MeshLab and commercial package Innovmetric's Polyworks, allow a Tpose to be generated with a small amount (10-15 minutes) of processing by human and machine.

\subsection{Data Collection Phase}

\subsubsection{Step 1:}

Place markers on the subject's arms that will be towards the edge of the scan volume when the arms are extended. Usually this maker will be placed close to the elbow, however, this will vary based on the scanner volume and the length of the subject's arms. These markers will be utilized to find common points when multiple scan files will be merged together. In the laboratory, 3 dimensional stickers (Fig. 5) are utilized to have an excellent frame of reference when color data is not available or insufficient. While these markers are not necessary to perform the merge, they do provide for faster merge results.

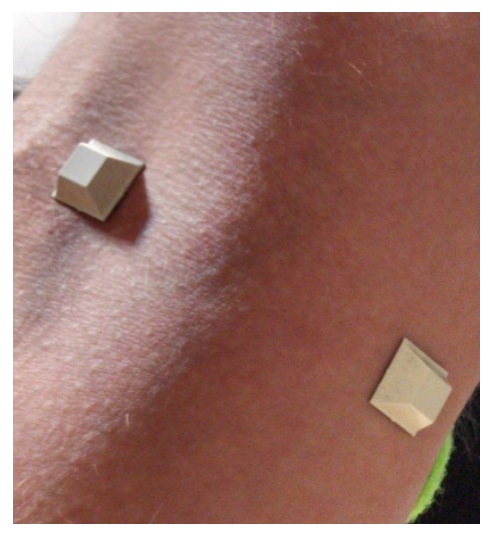

Fig. 5. 3D 'bumper' landmark. 


\subsubsection{Step 2:}

Place the subject in the center of the capture volume and scan while assuming the t-pose. The scanner will likely 'cut off' the subject's arms at approximately each elbow. Ensure that the landmarks placed in step 1 are visible.

\subsubsection{Step 3 \& 4:}

Move the subject to the left most portion of the volume and collect a scan of their right arm. Repeat the process in the rightward direction for the left arm. The three scans obtained in steps 2,3 , and 4 are sufficient to generate a vertex-mapped model.

\subsubsection{Step 5 (optional):}

Move the subject back to the center of the capture volume and take a scan with the entire body in the volume (most likely a CAESAR pose). This step is optional, but necessary if a texture mapped model is required. This is done to have a singular file in which all of the color data is stored.

\subsection{Data Processing Phase}

\subsubsection{Step 1:}

From the first three scans export the three T-pose meshes as 'vertex-mapped' scan files. Clean and hole-fill these files using a software package such as Innovmetric's Polyworks. For this purpose, we export the files as vertex-mapped .ply files. This allows scan manipulation software packages to edit meshes in color. Polyworks is commercial software, however, open source software, such as Meshlab can also be utilized. IMEdit is the Polyworks package that allows for scan file cleaning.

\subsubsection{Step 2:}

Import the newly cleaned files into IMAlign. Align, then merge the three files. Export the merged file. Using the 'N-Point Pairs Alignment' feature, which relies on a minimum of three points to align objects, select the 3D bumps placed around the elbow joint to align the two images (Fig. 6). Then use the same method for the opposite arm. Once the left and right arm images have been aligned properly, the IMMerge tool merges the three files together into one vertex-mapped .ply file (Fig. 7). At this stage, we have achieved one of our initial goals of having a 3D mesh file in a T-pose. Users may choose to stop processing at this point if they are satisfied with the use of a vertex-mapped file.

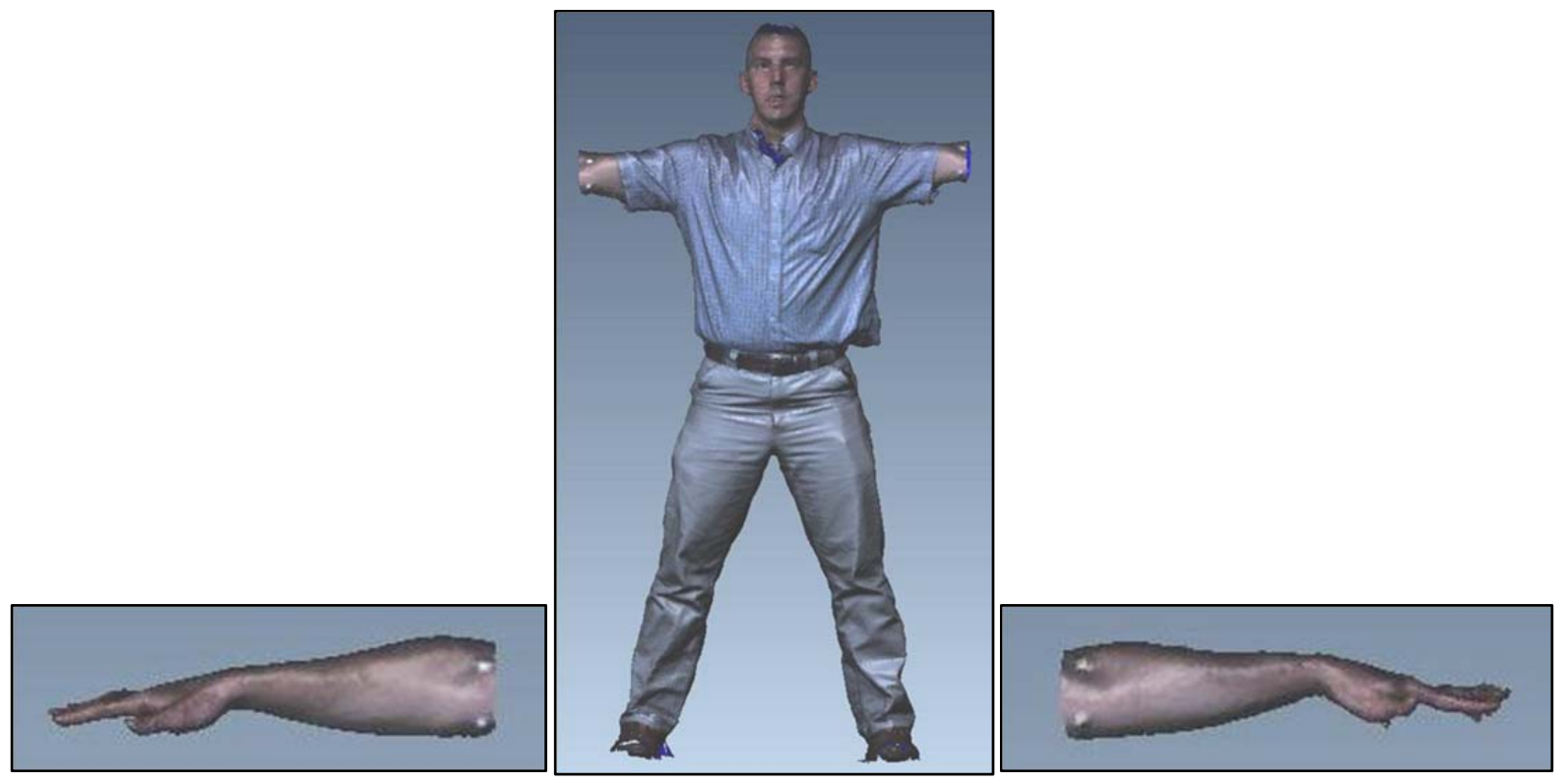

Fig. 6. Right Arm Scan (left); Body Scan with cut-off arms (middle); Left Arm Scan (right). 


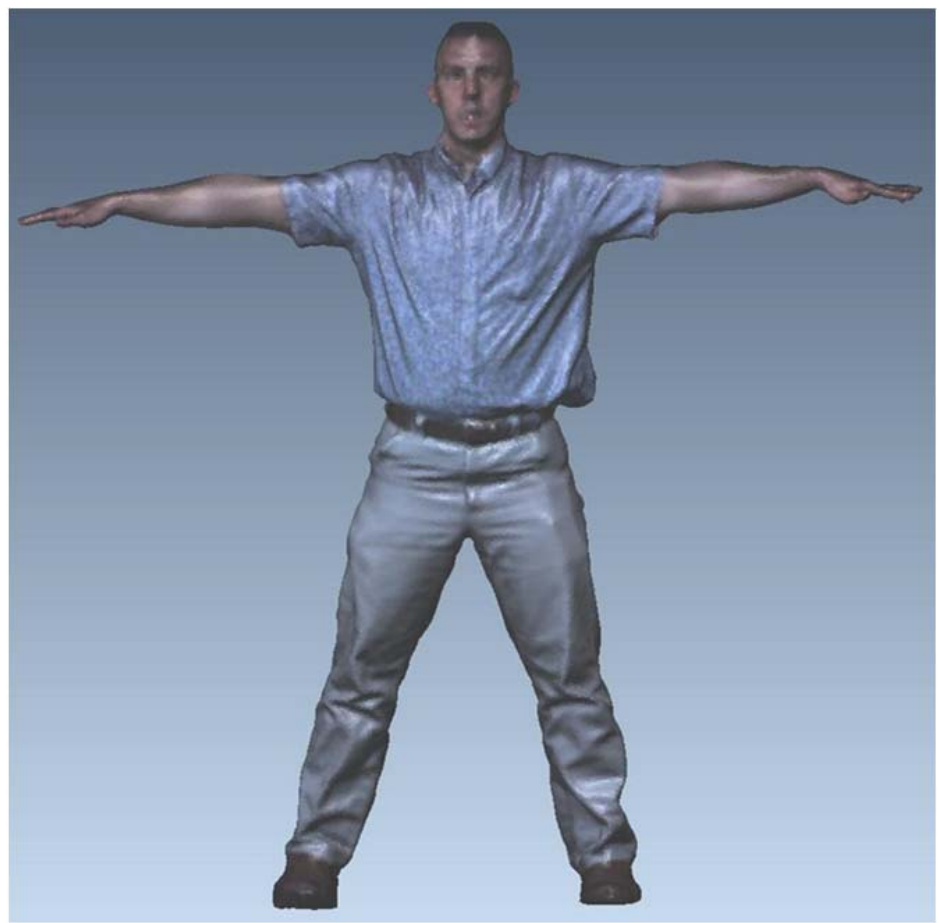

Fig. 7 Three files merged into one ply file.

\subsubsection{Step 3:}

Convert the newly merged file to an obj file. The obj format is preferred by animation software packages. However, at this point, the mesh file created is a 'vertex-mapped' file. When vertexmapped files are imported to most animation packages, the color information is lost from the file. A colorless geometry file is the result (Fig. 8). For animation purposes, a texture-mapped file, in which the texture of a mesh is mapped to a separate image file, is required. This necessitates several additional steps. Note: if a texture-mapped file is not required, the process ends at Step 2.

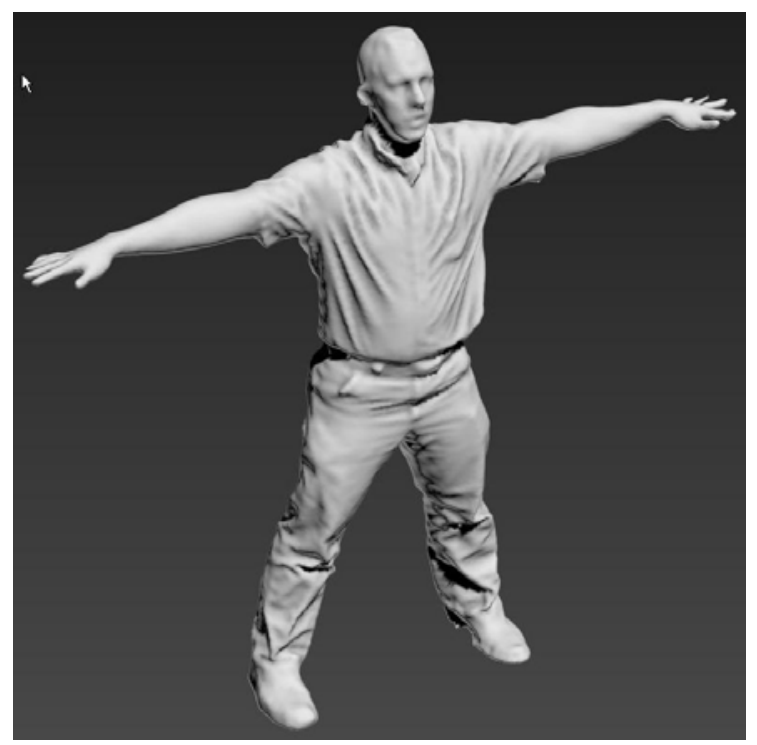

Fig. 8. Obj file with color missing.

\subsubsection{Step 4:}

Export a texture-mapped .obj file from the scanner in the original compact pose (likely a CAESAR Pose). When this mesh is exported from the scanner, the obj file is created, along with a specialized image file (in this case a .bmp) is created which wraps over the existing geometry. 


\subsection{Color Baking Phase}

At this point, we have two completed mesh files: a clean, hole-filled, T-pose which is colorless for animation purposes (Fig. 8) and a 'straight from the scanner' CAESAR pose file (obj) with color mapped to it (Fig. 9). Using animation software (in this case 3D Studio Max (3dsMax) by AutoDesk) the two files undergo a process we call color-baking. In this process the texture/color from (in this case) the CAESAR scan pose is baked onto the colorless geometry of the T-pose scan.

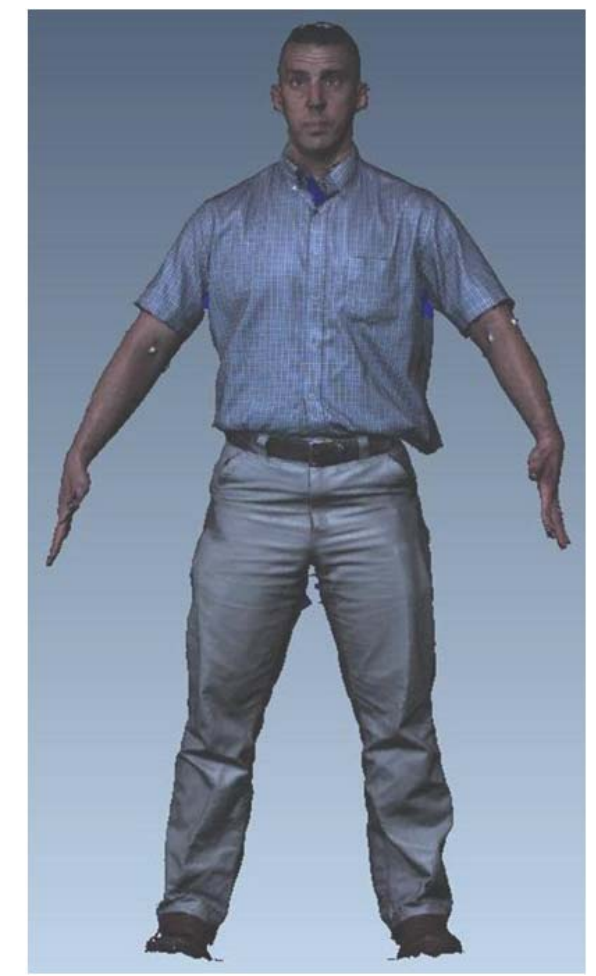

Fig. 9. CAESAR Pose Obj (Direct from Scanner).

\subsubsection{Step 1:}

Load a template model. Infoscitex has created a CAESAR template model (Fig. 10) that is used for this process. The model was created using a medium height individual and creating a slim exoskeleton using the 'Topology' function. This template model is a dense mesh designed to be morph-able into the shape of the subject in question. The template model includes an underlying associated biped model skeleton (Fig. 1).

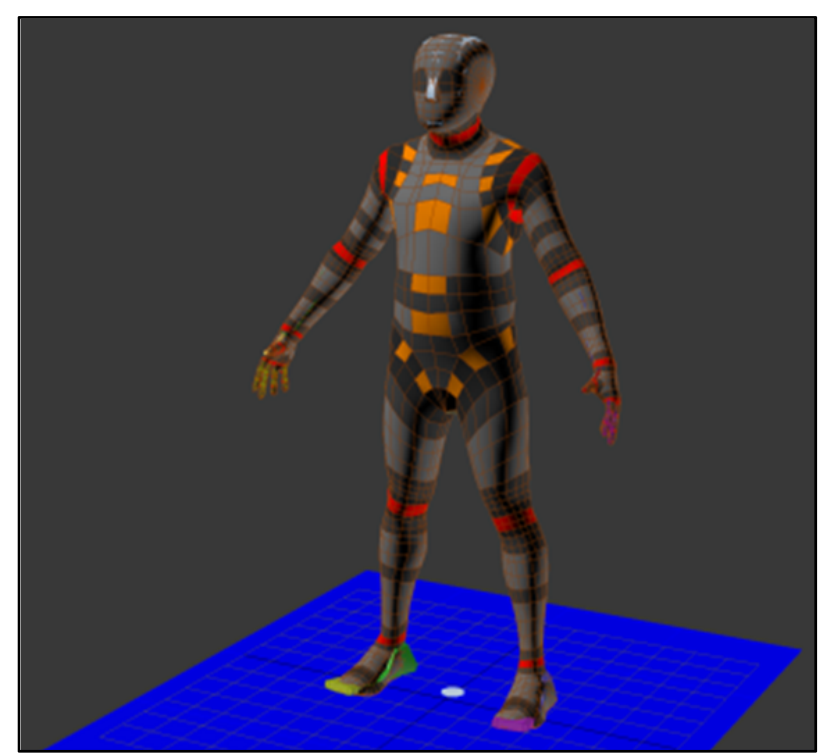

Fig. 10. CAESAR Template Model. 


\subsubsection{Step 2:}

Load the T-pose (colorless) scan into 3dsMax. Re-orient the template model to this pose. The skeletal portion of the template model (bones inside a mesh), is re-oriented to match the pose of the tpose scan and subsequently scaled to roughly match the subject height. Then, bones may require slight rotation or size adjustments as human body segments vary from person to person. Small adjustments must be made to correctly match the template model's skeleton to the actual subject's TPose exported in the data processing phase. (See Fig. 11)

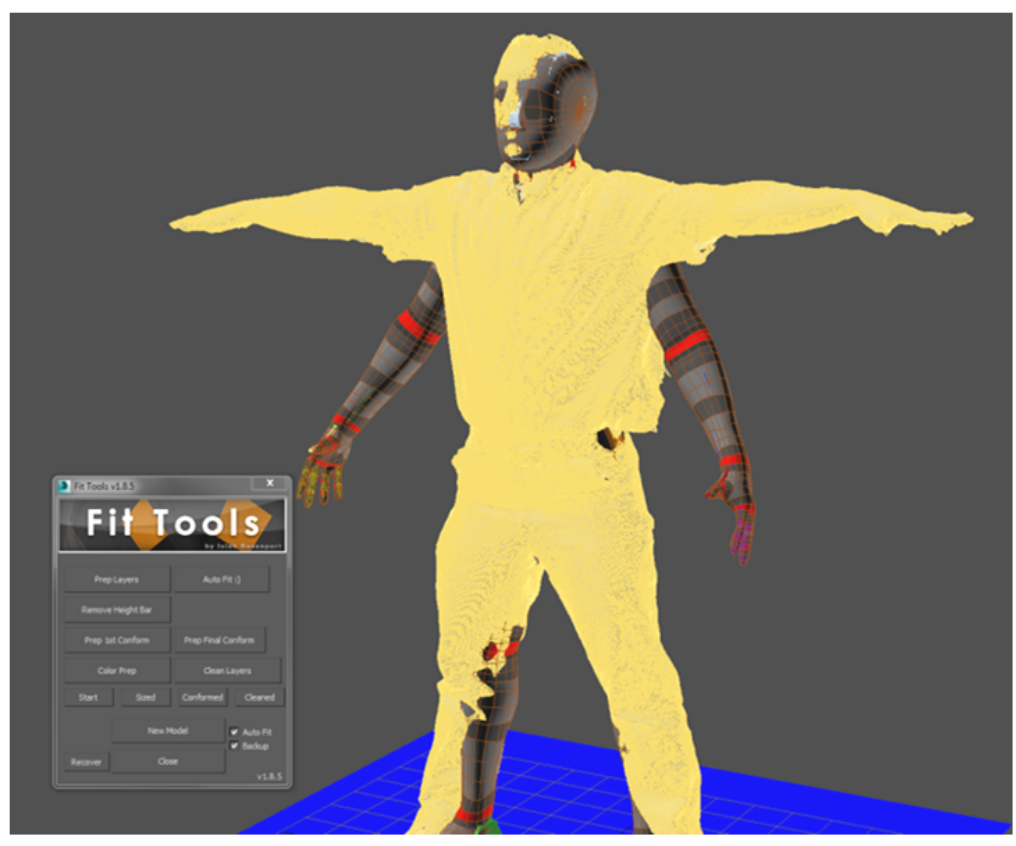

Fig. 11. Conforming template model to T-pose mesh.

\subsubsection{Step 3:}

Conform the template model to the scanned mesh. The imported mesh is likely too large in some areas, and too small in others. The template model must be adapted to match the actual scan. This is accomplished using the 'Conform' function (Fig. 12) to pull (or push) the vertices of the template model directly against the mesh of the subject In order to create a smooth mesh, often additional polygons must be created, then subsequently the 'Conform' function must be used once again. Once this is complete, the template model will match the hole-filled colorless t-pose.

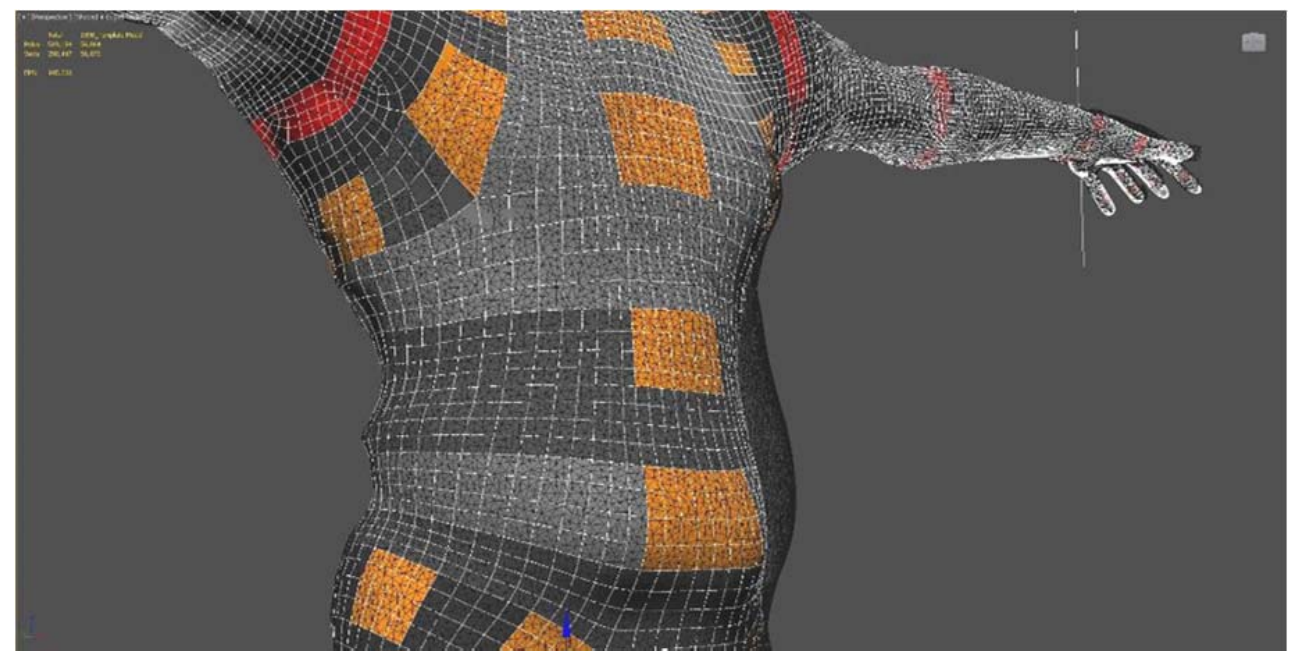

Fig. 12. Conforming template model to scan file. 


\subsubsection{Step 4:}

The template model is returned to match the CAESAR pose in order to import the color from that mesh. The texture-mapped CAESAR scan file is imported into 3dsMax. The template model human is then manipulated to match as closely as possible to the scan subject's posture (see Fig. 13). At this point the template model matches the original CAESAR Scan.

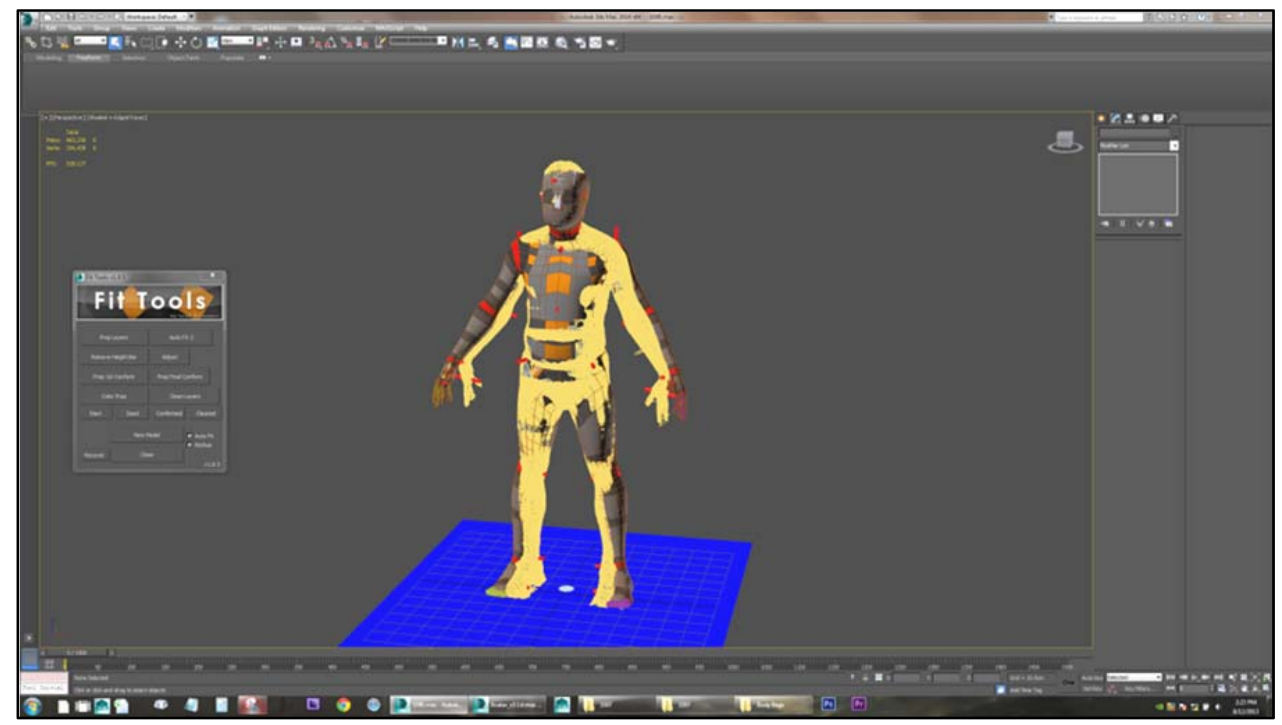

Fig. 13. Adjusting template model to CAESAR Scan.

\subsubsection{Step 5:}

Color-bake the CAESAR texture onto the template model. This is accomplished through the 3dsMax function 'Render To Texture'. The texture file from that scan (.bmp) will be 'baked' directly onto the template model. The model is then re-set to its T-pose state.

With these 5 steps complete, we are left with a texture-mapped obj file in a T-pose (Fig. 14).

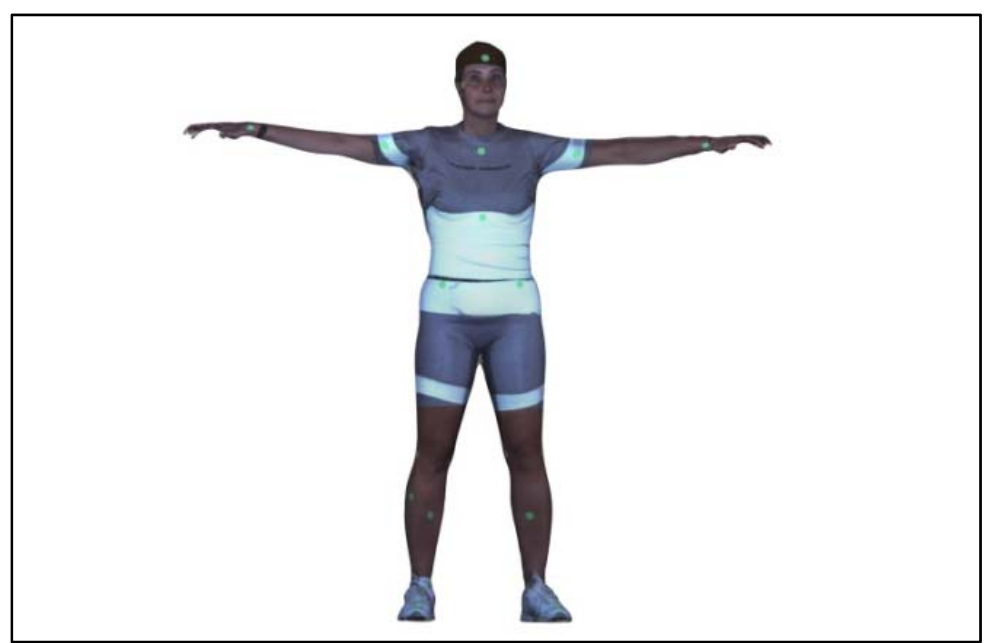

Fig. 14. Final texture mapped obj.

\section{Results}

As a result of this process, we are left with $3 D$ scan files that are useful to a large variety of researchers. The vertex-mapped ply files that are the result of the 'Data Processing' phase, provide a dense 3D mesh which could be useful for taking measurements, comparing 3D-shape, conducting Principle Component Analysis, etc. These files tend to be smaller, manipulate-able. They have the advantage of being easier to create and consist of only one file. The texture mapped obj file that is the result of the 'Color-Baking' phase is extremely useful for animators, though it can also be used for the wide variety of research purposes previously described. This file also has the advantage of being more visually pleasing to the average user, though it usually contains a fewer number of polygons, making the actual geometry slightly rougher. See Table 1 for a comparison of the two formats. 
Table 1. Comparison of Mesh File Formats.

\begin{tabular}{|c|c|c|}
\hline Aspect & Vertex-Mapped ply & Texture-Mapped obj \\
\hline Color/texture & Mapped to each vertex. & $\begin{array}{l}\text { In a separate file, wrapped } \\
\text { around the underlying } \\
\text { geometry. }\end{array}$ \\
\hline Number of files & 1 & $\begin{array}{l}2 \text { or } 3 \text {. The initial obj file, plus } \\
\text { the texture file, and material file } \\
\text { (if applicable). }\end{array}$ \\
\hline Physical File Size & Typically smaller & $\begin{array}{l}\text { Larger when all files are } \\
\text { considered. }\end{array}$ \\
\hline Mesh Appearance & $\begin{array}{l}\text { Color is limited to each vertex, } \\
\text { so it can look rough. }\end{array}$ & $\begin{array}{l}\text { Color is wrapped around the } \\
\text { mesh, making it look visually } \\
\text { stunning. }\end{array}$ \\
\hline Geometry & Generally smooth. & $\begin{array}{l}\text { Typically geometry is reduced, } \\
\text { to minimize file size. With color } \\
\text { overlay, geometry is not as } \\
\text { necessary. }\end{array}$ \\
\hline Ease of creation & $\begin{array}{l}\text { Medium. For a T-pose, it is still } \\
\text { necessary to merge files. } \\
\text { However, Color-baking process } \\
\text { is not required. }\end{array}$ & $\begin{array}{l}\text { Hard. Color baking process is } \\
\text { not trivial and can require } \\
\text { additional commercial software } \\
\text { packages. }\end{array}$ \\
\hline Able to be used in animation & No & Yes \\
\hline Close up of Fine Detail Image & Fig. 7 & Fig. 14 \\
\hline
\end{tabular}

\section{Conclusion}

The methodology developed by Infoscitex, allows for current generation 3D scanners to create meshes in poses deemed desirable by the largest majority of 3D scan users. As a result of this process, a very accurate T-pose mesh can be created with commercial software and a standard volume human scanner. Three scans must be taken of a given subject to generate a vertex-mapped file and an additional scan is required to generate a texture-mapped file.

\section{Acknowledgement}

This work is supported by the Human Signatures Branch of the Human-Centered Intelligence, Surveillance and Reconnaissance (ISR) Division, Human Effectiveness Directorate, United States Air Force Research Laboratory. The findings and conclusions in this report/presentation have not been formally disseminated by the Air Force and should not be construed to represent any agency determination or policy. This paper is approved for public release and distribution is unlimited. 88ABW-2013-3855 (27 Aug 2013).

\section{References}

1. Robinette, K., Blackwell, S., Daanen, H., Flemming, S., Boehmer, M., Brill, T., Hoeferlin, D., and Burnsides, D. (2002) "Civilian American and European Surface Anthropometry Resource (CAESAR), Final Report, Volume 1: Summary", AFRL-HE-WP-TR-2002-0169, Air Force Research Laboratory, Human Effectiveness Directorate, Bioscience and Protection Division, 2800 Q Street, Wright Patterson AFB OH 45433-7942.

2. "Topology Freeform." 3ds Max Help: Extension for Autodesk 3ds Max 2013. n.p., n.d., August 15, 2013. <http://docs.autodesk.com/3DSMAX/15/ENU/3ds-Max-Help/index.html>

3. "Conform Options Panel." 3ds Max Help: Extension for Autodesk 3ds Max 2013. n.p., n.d., August 15, 2013. <http://docs.autodesk.com/3DSMAX/15/ENU/3ds-Max-Help/index.html>

4. "Render to Texture." 3ds Max Help: Extension for Autodesk 3ds Max 2013. n.p., n.d., August 15, 2013. <http://docs.autodesk.com/3DSMAX/15/ENU/3ds-Max-Help/index.html> 